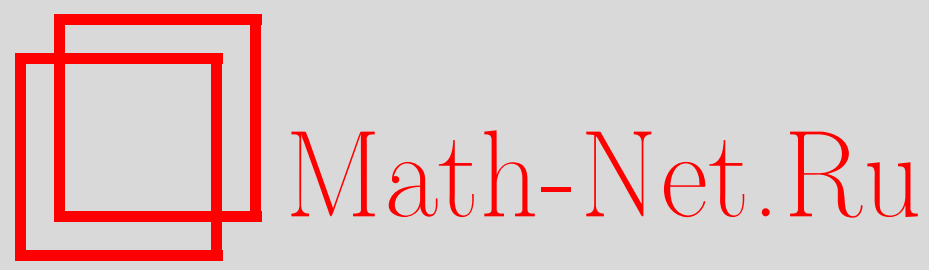

B. K. Белошапка, Инварианты CR-многообразий, связанные с касательной квадрикой, Матем. заметки, 1996, том 59, выпуск 1, 42-52

DOI: https://doi.org/10.4213/mzm1692

Использование Общероссийского математического портала MathNet.Ru подразумевает, что вы прочитали и согласны с пользовательским соглашением

http://www . mathnet.ru/rus/agreement

Параметры загрузки:

IP : 54.197 .130 .99

26 апреля 2023 г., 15:12:14

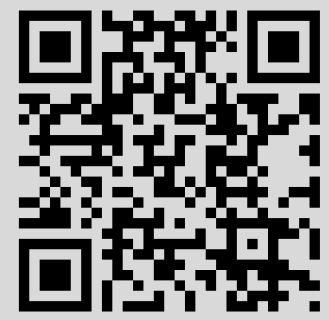




\section{ИНВАРИАНТЫ CR-МНОГООБРАЗИЙ, СВЯЗАННЫЕ С КАСАТЕЛЬНОЙ КВАДРИКОЙ}

\section{В.К. Белошапка}

\section{1. Введение}

Рассмотрим росток $M$ гладкой вещественной поверхности в начале координат комплексного линейного пространства размерности $N \geqslant 2$. Тогда в некоторой окрестности нуля $M$ можно задать системой соотношений

$$
\varphi^{p}=0, \quad p=1, \ldots, k \quad(1 \leqslant k \leqslant N-1) .
$$

Если $\{\operatorname{grad} \varphi(0), p=1, \ldots, k\} \mathbb{C}$-линейно независимы, то после комплексно-линейного преобразования уравнение $M$ можно записать в виде $\operatorname{Im} W=F(Z, \bar{Z}, \operatorname{Re} W) ; Z=\left(Z^{1}, \ldots, Z^{n}\right), W=\left(W^{1}, \ldots, W^{k}\right), N=n+k$, $F$ - гладкое отображение окрестности нуля в $\mathbb{C}^{n} \times \mathbb{R}^{k}$ в $\mathbb{R}^{k}$, причем $F$ и $d F$ равны нулю при $Z=0, \operatorname{Re} W=0$. Среди таких многообразий имеются $(k, n)$-квадрики, т.е. поверхности вида

$$
\operatorname{Im} W=\langle Z, Z\rangle,
$$

где $\langle Z, Z\rangle$ - это эрмитова форма со значениями в $\mathbb{R}^{k}$ или, другими словами, набор из $k$ скалярных эрмитовых форм $\left(\langle Z, Z\rangle^{1}, \ldots,\langle Z, Z\rangle^{k}\right)$, $\langle Z, Z\rangle^{p}=H^{p} Z \cdot \bar{Z}, H^{p}$ - эрмитова матрица порядка $n, p=1, \ldots, k$. Имеется голоморфная (полиномиальная) замена координат [1], после выполнения которой уравнение $M$ примет вид

$$
\operatorname{Im} W=\langle Z, Z\rangle+\text { члены степени три и более, }
$$

т.е. можно обеспечить касание третьего порядка. В этом случае $(k, n)$-квадрика, обозначим ее через $Q$, назьвается касательной квадрикой к поверхности $M$. Отметим, что размерность $M$ равна $k+2 n, \operatorname{Im} W=0$ - уравнение касательной плоскости в начале координат, $W=0$ - уравнение комплексной касательной, т.е. $n$ - это размерность комплексной касательной, a $\langle Z, Z\rangle$ - форма Леви поверхности $M$ в начале координат.

Работа вьполнена при финансовой поддержке Российского фонда фундаментальных исследований, грант № 093-01-00225. 


\section{Приведем некоторые сведения о касательных квадриках.}

УТВеРЖДЕНИЕ [2], [3], [4]. Если $\operatorname{Aut}(M)$ - это группа биголоморфных в окрестности начала координат преобразований $\mathbb{C}^{n+k}$, переводящих росток многообразия $M$ в начале координат в себя, то (1.1) $\operatorname{dim} \operatorname{Aut}(M) \leqslant \operatorname{dim} \operatorname{Aut}(Q)$, где $Q-$ касательная квадрика; (1.2) $\operatorname{dim} \operatorname{Aut}(Q)$ конечна в том и только в том случае, если выполнены следующие два условия:

координаты формы $\langle Z, Z\rangle$ линейно независимы,

из $\langle e, z\rangle=0$ для всех $z$ следует, что $е=0$, m.е. $\bigcap_{1}^{k} \operatorname{Ker} H^{p}=0$. (Квадрика и форма, удовлетворяющие этим условиям называются невырожденными.)

(1.3) Пусть $\langle z, z\rangle$ невырождена, тогда алгебра голоморфных векторных полей на $Q-g(Q)$ состоит из полей $X=f \frac{\partial}{\partial z}+g \frac{\partial}{\partial w}$, где

$$
\begin{aligned}
& f=p+C z+a w+A(z, z)+B(z, w), \\
& g=q+s w+2 i\langle z, p+a w\rangle+r(w, w),
\end{aligned}
$$

все выражения полилинейны, причем

$$
\begin{gathered}
\operatorname{Im} q=0, \quad 2 \operatorname{Re}\langle C z, z\rangle=s\langle z, z\rangle, \quad\langle A(z, z), z\rangle=2 i\langle z, a\langle z, z\rangle\rangle, \\
\operatorname{Im} r=0, \quad \operatorname{Re}\langle B(z, u), z\rangle=r(\langle z, z\rangle, u), \quad \operatorname{Im}\langle B(z,\langle z, z\rangle), z\rangle=0 .
\end{gathered}
$$

(1.4) Вводя в $g(Q)$ естественную градуировку ( $z$ “весит" $1, w-2$, $\left.\frac{\partial}{\partial z}-(-1) u \frac{\partial}{\partial w}-(-2)\right)$, мы получаем разложение алгебры $g(Q)$ на однородние компоненты $g(Q)=g_{-2}+g_{-1}+g_{0}+g_{1}+g_{2}, g_{-2}+g_{-1}-$ это подалгебра, порожденная группой аффинных преобразований $\{z \rightarrow p+z$, $w \rightarrow q+w+2 i\langle z, p\rangle\}$, транзитивно действующей на $Q, \operatorname{dim} g_{-2}=k$, $\operatorname{dim} g_{-1}=2 n$. Подалгебра $g_{0}-$ это алгебра әруппь LAut $(Q)$ линейньх преобразований $Q$ вида $\{z \rightarrow C z, w \rightarrow s w$, где $\langle C z, C z\rangle=s\langle z, z\rangle\}$. При $k=1 \operatorname{dim} g_{0}=n^{2}+1, \operatorname{dim} g_{1}=2 n, \operatorname{dim} g_{2}=1$. При бо́льиих значениях $k$ набор размерностей зависит от квадрики, так для квадрики общего полохсения при $k=2 \operatorname{dim} g_{0}=n+1, \operatorname{dim} g_{1}=0, \operatorname{dim} g_{2}=0$, a при $3 \leqslant k \leqslant n^{2}-3 \operatorname{dim} g_{0}=2, \operatorname{dim} g_{1}=0, \operatorname{dim} g_{2}=0$.

(1.5) Положим

$$
\begin{aligned}
\Gamma(p) & =\left\{z \in \mathbb{C P}^{n-1}: \operatorname{rank}_{\mathbb{C}}\left(H^{1} z, \ldots, H^{k} z\right) \leqslant p\right\}, \\
\Gamma r(p) & =\left\{z \in \mathbb{R P}^{2 n-1}: \operatorname{rank}_{\mathbb{R}}\left(H^{1} z, \ldots, H^{k} z\right) \leqslant p\right\}, \\
\gamma(q) & =\left\{w \in \mathbb{C P}^{k-1}: \operatorname{rank}\left(w^{1} H^{1}+\cdots+w^{k} H^{k}\right) \leqslant q\right\}, \\
\gamma r(q) & =\left\{u \in \mathbb{R P}^{k-1}: \operatorname{rank}\left(u^{1} H^{1}+\cdots+u^{k} H^{k}\right) \leqslant q\right\} .
\end{aligned}
$$

Пусть имеются два CR-многообразия $M_{1}$ и $M_{2}$ типа $(k, n)$, чьи локальнье уравнения записань в форме (2) $и\langle z, z\rangle_{1},\langle z, z\rangle_{2}-$ их форми 
Леви соответственно, $f: M_{1} \rightarrow M_{2}$ - локальный CR-диффеоморфизм класса $C^{2}$ вида $z \rightarrow C z+\cdots, w \rightarrow s w+\cdots, C \in \mathrm{GL}(n, \mathbb{C}), s \in \operatorname{GL}(k, \mathbb{R})$. Тогда, если $\Gamma_{j}(p), \Gamma r_{j}(p), \gamma_{j}(q), \gamma r_{j}(q)$ - система, соответствующая $\langle z, z\rangle_{j}$, mo $C\left(\Gamma_{1}(p)\right)=\Gamma_{2}(p), C\left(\Gamma r_{1}(p)\right)=\Gamma r_{2}(p), s^{\prime}\left(\gamma_{1}(q)\right)=\gamma_{2}(q)$, $s^{\prime}\left(\gamma r_{1}(q)\right)=\gamma r_{2}(q)\left(s^{\prime}-\right.$ транспонированная $\left.s\right)$, т.е. эти системы множеств CR-инвариантны.

(1.6) Если из координатных матрии $H^{1}, \ldots, H^{k}$ нельзя составить невырожденной линейной комбиначии, то квадрика называется нульквадрикой. При этом многочлен $P_{H}(t)=\operatorname{det}\left(t^{1} H^{1}+\cdots+t^{k} H^{k}\right)$ равен нулю тождественно и

$$
\gamma(n-1)=\left\{w \in \mathbb{C P}^{k-1}: P_{H}(t)=0\right\}=\mathbb{C P}^{k-1} .
$$

\section{2. Проективные инварианты квадрики}

Квадрика является алгебраическим множеством, поэтому можно рассмотреть $\bar{Q}$ - замыкание в $\mathbb{C P}^{n+k}$. Если однородные координаты в $\mathbb{C P}^{n+k}(t, z, w), t \in \mathbb{C}^{1}, z \in \mathbb{C}^{n}, w \in \mathbb{C}^{k}$ ввести так, что $Z=z / t, W=w / t$, то уравнение $\bar{Q}$ будет выглядеть следующим образом $\operatorname{Im} w \bar{t}=\langle z, z\rangle$. Все точки аффинной части квадрики являются неособыми, как это видно из уравнения (1), более того, аффинная часть квадрики является аффинно-однородной. Если $k=1$, то $\bar{Q}$ остается неособой и проективно однородной. Наблюдение, на котором основана данная работа, состоит в том, что при $k>1$ это не так. На бесконечности у квадрики появляются особенности. Простейшая характеристика особой точки - это размерность касательного пространства в ней. Ниже мы осуществляем соответствующее построение.

Пусть $e_{j}(t, z, w)=\operatorname{grad}\left(-\operatorname{Im} w^{j}+\langle z, z\rangle^{j}\right)$. Положим для $(t, z, w) \in \bar{Q}$

$$
\begin{aligned}
\sigma r(t, z, w) & =\operatorname{rank}_{\mathbb{R}}\left(e_{1}(t, z, w), \ldots, e_{k}(t, z, w)\right), \\
\sigma c(t, z, w) & =\operatorname{rank}_{\mathbb{C}}\left(e_{1}(t, z, w), \ldots, e_{k}(t, z, w)\right),
\end{aligned}
$$

т.е. это вещественный и комплексный ранги системы градиентов.

ЛЕмма 1. Величины $\sigma r$ и бс сохраняются при регулярных комплексных отображениях.

ДокаЗАтЕльство. Гиперповерхности $\left\{-\operatorname{Im} w^{j} \bar{t}+\langle z, z\rangle^{j}=0\right\}$ в афффинной части $\mathbb{C P}^{n+k}$ пересекаются трансверсально, поэтому определяющие их многочлены порождают идеал множества $Q$ [5], а, следовательно, и идеал множества $\bar{Q}$. Таким образом, касательное пространство к $\bar{Q}$ задается уравнениями $d\left(-\operatorname{Im} w^{j} \bar{t}+\langle z, z\rangle^{j}\right)=0, j=1, \ldots, k$. Размерность касательного пространства инварианта при регулярных изоморфизмах. А 
$\delta r$ - это разница между размерностью объемлющего пространства и размерностью касательного. Поэтому $\delta r$ не может измениться. Аналогичное рассуждение, проведенное для комплексной части касательного пространства, доказывает инвариантность $\delta c$.

В результате мы получаем две стратификации $\bar{Q}$ на точки с различными значениями $\delta r$ и $\delta c$, которые на $Q$ тривиальны (вся аффинная часть попадает в страты максимальньх рангов), а на бесконечности характеризуют особые точки. Пусть $p=0,1, \ldots, k$, положим

$$
\begin{aligned}
& \delta r(p)=\{(t, z, w) \in \bar{Q}: \delta r(t, z, w) \leqslant p\}, \\
& \delta c(p)=\{(t, z, w) \in \bar{Q}: \delta c(t, z, w) \leqslant p\} .
\end{aligned}
$$

УТВЕРЖДЕНИЕ 2. Имеют место вложения:

(2.1) $\delta r(0) \subset \delta r(1) \subset \cdots \subset \delta r(k-1) \subset \delta r(k)=\bar{Q}$,

$\delta c(0) \subset \delta c(1) \subset \cdots \subset \delta c(k-1) \subset \delta c(k)=\bar{Q}$,

(2.2) $\delta r(p) \subset \delta c(p) \subset \delta r(2 p)$,

(2.3) $Q \subset \delta r(k) \backslash \delta r(k-1), Q \subset \delta c(k) \backslash \delta c(k-1)$.

Утверждение вполне очевидно.

УТВЕРЖДЕНИЕ 3. Пусть имеются два CR-многообразия $M_{1}$ и $M_{2}$ mипа $(k, n),\langle Z, Z\rangle_{1},\langle Z, Z\rangle_{2}$ - их формы Леви соответственно, $f: M_{1} \rightarrow M_{2}$ - локальный CR-диффеоморфизм класса $C^{2}$ вида

$$
Z \rightarrow C Z+\cdots, W \rightarrow s W+\cdots, \quad C \in \mathrm{GL}(n, \mathbb{C}), s \in \mathrm{GL}(k, \mathbb{R}) .
$$

Тогда:

(3.1) $\langle C Z, C Z\rangle_{2}=s\langle Z, Z\rangle_{1} u$, тем самым, $Z \rightarrow C Z, W \rightarrow$ sW есть линейный изоморфизм касательных квадрик,

(3.2) проективизация этого линейного отображения $t \rightarrow t, z \rightarrow C z$, $w \rightarrow$ sw переводит систему множеств $\{\delta c(p)\}_{1}$ в $\{\delta c(p)\}_{2}, a\{\delta r(p)\}_{1}$ $\boldsymbol{\theta}\{\delta r(p)\}_{2}$.

ДоКАЗАТЕЛЬСтво. (3.1) Соотношение есть запись CR-инвариантности формы Леви.

(3.2) Проективное отображение сохраняет размерность касательного пространства.

Ввиду (2.3) при изучении особых точек можно ограничиться бесконечно удаленной плоскостью $\{t=0\}$. Бесконечно удаленные точки $\bar{Q}$ задаются следуюшими уравнениями

$$
Q^{\infty}=\bar{Q} \backslash Q=\{t=0,\langle z, z\rangle=0\} .
$$

Уравнения касательной имеют следующий вид

$$
\operatorname{Re}(i w d \bar{t}-i t d \bar{w}+2\langle z, d z\rangle)=0 .
$$


Если обозначить через $x \cdot \bar{y}$ стандартную эрмитову форму $x^{1} \bar{y}^{1}+\cdots+x^{n} \bar{y}^{n}$ в $\mathbb{C}^{n}$, а через $H^{p}$ - матрицу формы $\langle z, z\rangle^{p}$, то уравнение можно записать таK:

$$
\operatorname{Re}\left(-i \bar{w}^{p} d t+i \bar{t} d w^{p}+2 H^{p} \bar{z}-d z\right)=0, \quad p=1, \ldots, k,
$$

а уравнения комплексной касательной так:

$$
-i \bar{w}^{p} d t+i \bar{t} d w^{p}+2 H^{p} \bar{z}-d z=0, \quad p=1, \ldots, k .
$$

Если обозначить $h^{p q} z q$-ю координату вектора $H^{p} z$, то получим следующее утверждение.

УТВЕРЖДЕНИЕ 4. Величины $\delta c(t, z, w) u \delta r(t, z, w)$ - это ранг комплексной матрицы размера $k \times(n+k+1)$ вида $H=(i w, 2 H z,-i t E)$, где $E$ - это единичная матрича размером $k \times k$

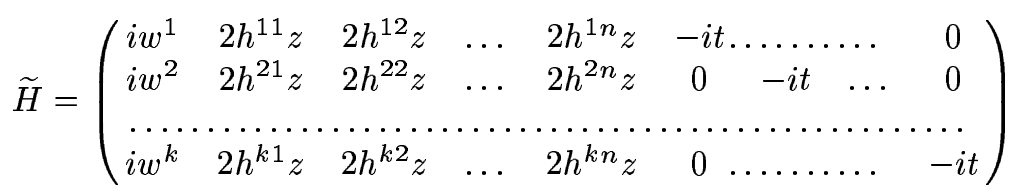

и ранг вещественной матрицы размера $k \times 2(n+k+1)$, полученной из первой отделением в каждом столбце действительной и мнимой части.

Величины $\delta c(0, z, w)$ и $\delta r(0, z, w)$ аналогичньм образом связаны с матрицей размера $k \times(n+1)$ вида $H^{\infty}=(i w, 2 H z)$.

СлЕДСТВИЕ 1. $\delta r(p)$ u $\delta c(p)$ - это пересечение квадрики с некоторыми проективными множсествами, которые задаются условиями на ранг матриив $\widetilde{H}$.

УТВЕРЖДЕНИЕ 5.

(5.1) $\delta r(0)=\delta c(0)=\varnothing$,

(5.2) если $p>\min (k, n+1), p \neq k, \operatorname{mo} \delta c(p)=\varnothing$,

(5.3) если $p>\min (k, 2 n+1), p \neq k, \operatorname{mo} \delta r(p)=\varnothing$,

(5.4) бесконечно удаленная часть всякой квадрики содержит $\mathbb{C P}^{k-1}$, состоящее из точек вида $\left\{(0,0, w): w \in \mathbb{C P}^{k-1}\right\}$, которое содержится в $\delta c(1)$ и в $\delta r(2)$, причем $\left\{(0,0, w): w \in \mathbb{C P}^{k-1}, \operatorname{Im} w^{p} \bar{w}^{q}=0\right\} \subset \delta r(1)$.

ДокАЗАТЕльСТво. (5.1) Если ранги равны нулю, товсеэлементы равны нулю. Это означает, что $w=0$ и $H^{p} z=0$ для всех $p$. Но в силу невырожденности формы $\langle z, z\rangle$ отсюда следует, что $z=0$ и $t=0$, так как иначе точка имеет ранг $k$.

(5.2) Если $p \neq k$, то $\delta c(p)$ не содержит конечных точек, но в этом случае ранг - это ранг матрицы $H^{\infty}$ размером $k \times(n+1)$.

(5.3) Аналогично.

(5.4) В этом случае $H^{\infty}=(i w, 0)$. 
ПримеР 1. Рассмотрим случай гиперквадрики $(k=1)$. Тогда $G=$ $\left(i w, 2 h^{1} z, \ldots, 2 h^{n} z,-i t\right)$, при этом, в силу $(5.1), \delta c(0)=\delta r(0)=\varnothing$, т.е. $\bar{Q}=\delta c(1)=\delta r(1)$. Все точки неособые. Группа проективных преобразований $\mathbb{C P}^{n+1}$ транзитивно действует на $\bar{Q}$.

ПримеР $2(k=2)$. В этом случае

$$
\begin{aligned}
& H^{\infty}=\left|\begin{array}{ll}
i w^{1} & 2 H^{1} z \\
i w^{2} & 2 H^{2} z
\end{array}\right|, \\
& \delta c(1)=\left\{\left\{(0,0, w): w \in \mathbb{C P}^{1}\right\} \cup\{(0, z, w): z \in \Gamma(1),\right.
\end{aligned}
$$

$$
\left.\left.l \cdot w=0, l=\left(l^{1}, l^{2}\right) \in \gamma(n-1)\right\}\right\} \cap Q^{\infty},
$$

т.е. $z$ - это собственньй вектор задачи $l^{1} H^{1} z+l^{2} H^{2} z=0$, a $l$ - соответствуюшее собственное значение.

$$
\begin{array}{r}
\delta r(1)=\left\{\left\{(0,0, w): w \in \mathbb{C P}^{1}, \operatorname{Im} w^{1} \bar{w}^{2}=0\right\} \cup\{(0, z, w): z \in \Gamma r(1),\right. \\
\left.\left.l \cdot w=0, l=\left(l^{1}, l^{2}\right) \in \gamma r(n-1)\right\}\right\} \cap Q^{\infty},
\end{array}
$$

т.е. $z$ - это собственньй вектор задачи $l^{1} H^{1} z+l^{2} H^{2} z=0$, a $l$ - соответствуюшее вешественное собственное значение.

Из примера видно, что введенные здесь инвариантные множества $\delta c(p)$ и $\delta r(p)$ связаны с системой инвариантов $\Gamma(p), \Gamma r(p), \gamma(q), \gamma r(q)$. Однако инвариантные множества $\Gamma(p)$ и $\Gamma r(p)$ расположены в пространстве переменных $z$, множества $\gamma(q)$ и $\gamma r(q)$ - в пространстве переменных $w$ и $u$, тог да как уравнения, задаюшие множества $\delta c(p)$ и $\delta r(p)$ связывают обе групшы переменных.

ПРимеР 3. Рассмотрим в качестве примера ситуацию $k=n=2$. Как известно [6], имеются всего три попарно неэквивалентные $(2,2)$-квадрики

$$
\left|\begin{array}{l}
2 \operatorname{Re} z^{1} \bar{z}^{2} \\
2 \operatorname{Im} z^{1} \bar{z}^{2}
\end{array}\right|,\left|\begin{array}{l}
\left|z^{1}\right|^{2} \\
\left|z^{2}\right|^{2}
\end{array}\right|, \quad\left|\begin{array}{c}
\left|z^{1}\right|^{2} \\
2 \operatorname{Re} z^{1} \bar{z}^{2}
\end{array}\right|
$$

В восьмимерном вещественном пространстве $(2,2)$-квадрик квадрики первого и второго типа - это две области, разделенные гиперповерхностью, состоящей из квадрик третьего типа.

Первьй тип:

$$
\begin{aligned}
& \delta c(1)=\left\{\left(0,0,0 ; w^{1}, w^{2}\right)\right\} \cup\{(0 ; 0, z ; w, i w)\} \cup\{(0 ; 0, z ; w,-i w)\}, \\
& \delta r(1)=\left\{\left(0 ; 0,0, w^{1}, w^{2}\right), \text { где } \operatorname{Im} w^{1} \bar{w}^{2}=0\right\} .
\end{aligned}
$$

Второй тип:

$$
\begin{aligned}
& \delta c(1)=\left\{\left(0 ; 0,0, w^{1}, w^{2}\right)\right\}, \\
& \delta r(1)=\left\{\left(0 ; 0,0, w^{1}, w^{2}\right), \text { где } \operatorname{Im} w^{1} \bar{w}^{2}=0\right\} .
\end{aligned}
$$

Третий тип:

$$
\begin{aligned}
& \delta c(1)=\left\{\left(0 ; 0,0 ; w^{1}, w^{2}\right)\right\} \cup\{(0 ; 0, z ; 0, w)\}, \\
& \delta r(1)=\left\{\left(0 ; 0,0, w^{1}, w^{2}\right), \text { где } \operatorname{Im} w^{1} \bar{w}^{2}=0\right\} \cup\{(0 ; 0, z ; 0, w)\} .
\end{aligned}
$$




\section{3. Проективные автоморфизмы квадрики}

Выписанные вьше системы подмножеств $\bar{Q}$ инвариантны по отношению как к аффинным, так и проективньм автоморфизмам. Группа линейных автоморфизмов $\operatorname{LAut}(Q)$ состоит из преобразований вида

$$
t \rightarrow t, \quad z \rightarrow C z, \quad w \rightarrow s w,
$$

с соотношением $\langle C z, C z\rangle=s\langle z, z\rangle$ эта группа является подгруппой в группе $\operatorname{PAut}(Q)$ проективньг преобразований $\bar{Q}$. $\operatorname{PAut}(Q)$ при $k=1$ исчерпывает всю группу автоморфизмов $\operatorname{Aut}(Q)$. При $k>1$ это, вообще говоря, не так. Например, при $k=2 \operatorname{Aut}(Q)$ может состоять из квадратично-рациональных преобразований, хотя для квадрики общего положения группа содержит лишь линейные преобразования [7]. Наша ближайшая цель - получить описание $\mathrm{PAut}_{0}(Q)$ - групш дробно-линейных преобразований $Q$, сохраняюших начало координат в $\mathbb{C}^{n+k}$. Всякое такое преобразование имеет вид

$$
Z \rightarrow \frac{C(Z+a W)}{1-(\beta Z+\gamma W)}, \quad W \rightarrow \frac{s(W+b Z)}{1-(\beta Z+\gamma W)}
$$

Записьвая, что (3) есть автоморфизм квадрики, получаем

$$
\operatorname{Re}(i(1-(\overline{\beta Z+\gamma W})) s(W+b Z)+\langle C(Z+a W), C(Z+a W)\rangle)=0
$$

при $W=U+i\langle Z, Z\rangle$.

Отделяя однородные компоненты (4), получаем $b=0$,

$$
\begin{gathered}
\langle C Z, C Z\rangle=s\langle Z, Z\rangle, \quad \text { т.e. }(C, s) \in \operatorname{LAut}(Q), \\
\beta Z U=2 i\langle Z, a U\rangle,
\end{gathered}
$$

если $\operatorname{Re} \gamma(U)=r(U), \operatorname{Im} \gamma(U)=\sigma(U)$, то

$$
\begin{gathered}
\sigma(U) U=\langle a U, a U\rangle, \\
r(\langle Z, Z\rangle) U-r(U)\langle Z, Z\rangle=2 \operatorname{Im}\langle a\langle Z, Z\rangle, a U\rangle .
\end{gathered}
$$

При $k=1$ эти соотношения принимают вид

$$
\beta Z=2 i\langle Z, a\rangle, \quad \sigma(U)=\langle a, a\rangle U,
$$

и мы получаем известную формулу

$$
\begin{gathered}
Z \rightarrow \frac{C(Z+a W)}{1-(2 i\langle Z, a\rangle+(r+i\langle a, a\rangle) W)}, \\
W \rightarrow \frac{s W}{1-(2 i\langle Z, a\rangle+(r+i\langle a, a\rangle) W)} .
\end{gathered}
$$


При произвольном $k$ запишем (5) покоординатно

$$
\begin{aligned}
& \beta Z U^{1}=2 i\left\langle Z, a_{1}\right\rangle^{1} U^{1}+\cdots+2 i\left\langle Z, a_{k}\right\rangle^{1} U^{k}, \\
& \cdots \cdots \cdots \cdots \cdots \cdots \cdots \cdots \cdots \cdots \cdots \cdots \cdots \cdots \cdots \cdots \cdots \cdots \cdots+2 i\langle Z, a\rangle_{k}^{k} U^{k} \\
& \beta Z U^{k}=2 i\langle Z, a\rangle_{1}^{k} U^{1}+\cdots+
\end{aligned}
$$

где $a_{p}-p$-й столбец матрицы $a$. То есть $a_{p} \in \operatorname{Ker} H^{q}$ при $p \neq q, H^{p} a_{p}=\beta$, $\beta Z=2 i Z \cdot \bar{\beta}$, где $\beta \in \mathbb{C}^{n}$. Таким образом, условие существования ненулевого решения вьглядит так

$$
\bigcap_{p=1}^{k} H^{p}\left(\bigcap_{q} \operatorname{Ker} H^{q}\right) \neq 0 \quad \text { или } \quad H^{p} a_{q}=\delta_{q}^{p} \beta,
$$

где $\delta_{q}^{p}$ - символ Кронекера и $\beta \neq 0$. Это условие возникало в работе Палинчак [8], как условие того, что из $c$-жесткости $\left(\operatorname{dim} g_{0}=2\right)$ следует $a$-жесткость $\left(g_{1}=0\right)$.

Оставшиеся соотношения (6) и (7) дают следуюшие условия на векторы $a_{1}, \ldots, a_{k}$.

$$
2 i\left\langle a_{p}, a_{q}\right\rangle=\gamma_{q} e_{p}-\bar{\gamma}_{p} e_{q},
$$

откуда получаем $\gamma_{q}=2 i H^{p} a_{p} \cdot \bar{a}_{q}$. Но в силу эрмитовости $H^{p} a_{p}-\bar{a}_{q}=$ $a_{p}-\overline{H^{p}} a_{q}=0$, т.е. $\gamma_{p}=0$. Таким образом, $\left\langle a_{p}, a_{q}\right\rangle=0$ для всех $p$ и $q$. Из этих соотношений в (9) не содержатся лишь $H^{p} a_{p}-\bar{a}_{p}=0$, т.е. $a_{p}-\bar{\beta}=0$. В итоге получаем

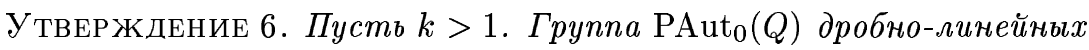
преобразований $Q$, сохраняющих начало координат, состоит из преобразований вида

$$
Z \rightarrow \frac{C(Z+a W)}{1-2 i Z \bar{\beta}}, \quad W \rightarrow \frac{s W}{1-2 i Z \bar{\beta}}
$$

əде $(C, s) \in \operatorname{LAut}(Q)$, причем

$$
H^{p} a_{q}=\delta_{q}^{p} \beta, \quad a_{p}-\bar{\beta}=0 \quad \text { для всех } p \text { и } q .
$$

ЗАмЕчАнИЕ. Отсутствие параметра $\gamma$, т.е. соотношение $\gamma=0$ означает, что при $k>1$ в отличие от случая $k=1$, все проективные автоморфизмы являются $b$-жесткими $\left(g_{2}=0\right)$.

СлЕДСТВИЕ 2. Нелинейные проективные автоморфизмы могут быть лишь у нуль-квадрик.

ДокАЗАТЕЛЬСТво. Иначе нарушается условие (10). 
ПримеР 4. Среди нуль-квадрик имеются квадрики, такие, что все их автоморфизмы - дробно-линейные преобразования. Шевченко [9] построила классификацию квадрик коразмерности два. Всякая нуль-квадрика, имеющая нелинейные автоморфизмы при $k=2$ задается парой форм следуюшего вида

$$
\begin{aligned}
& \langle Z, Z\rangle^{1}=\sum_{j=1}^{s} \operatorname{Re} Z^{3 j-2} \bar{Z}^{3 j-1}+[\widetilde{Z}, \widetilde{Z}]^{1}, \\
& \langle Z, Z\rangle^{2}=\sum_{j=1}^{s} \operatorname{Re} Z^{3 j-2} \bar{Z}^{3 j}+[\widetilde{Z}, \widetilde{Z}]^{2}
\end{aligned}
$$

где $[\widetilde{Z}, \widetilde{Z}]^{j}$ - эрмитовы формы от $\widetilde{Z}=\left(Z^{3 s+1}, \ldots, Z^{n}\right)$, такие, что из их матриц $\widetilde{H}^{1}$ и $\widetilde{H}^{2}$ можно составить невырожденную линейную комбинацию. Ежов и Шмальц [10], пользуясь классификацией Шевченко, вьписали формулы для нелинейных преобразований квадрики коразмерности два. В частности, все нелинейные автоморфизмы данной квадрики - это дробно-линейные преобразования вида

$$
\begin{aligned}
Z^{3 p-2} & \rightarrow Z^{3 p-2} /\left(1-2 i \sum_{1}^{s} \bar{a}^{j} Z^{3 j-2}\right) \\
Z^{3 p-1} & \rightarrow\left(Z^{3 p-1}+a^{p} W^{1}\right) /\left(1-2 i \sum_{1}^{s} \bar{a}^{j} Z^{3 j-2}\right), \\
Z^{3 p} & \rightarrow\left(Z^{3 p}+a^{p} W^{2}\right) /\left(1-2 i \sum_{1}^{s} \bar{a}^{j} Z^{3 j-2}\right), \quad p=1, \ldots, s ; \\
Z^{p} & \rightarrow Z^{p} /\left(1-2 i \sum_{1}^{s} \bar{a}^{j} Z^{3 j-2}\right), \quad p=3 s+1, \ldots, n, \\
W^{1} & \rightarrow W^{1} /\left(1-2 i \sum_{1}^{s} \bar{a}^{j} Z^{3 j-2}\right), \\
W^{2} & \rightarrow W^{1} /\left(1-2 i \sum_{1}^{s} \bar{a}^{j} Z^{3 j-2}\right) .
\end{aligned}
$$

\section{4. Преобразования квадрики как подгруппа группы Кремоны}

Туманов доказал [11], что всякое CR-отображение произвольной невырожденной $(k, n)$-квадрики в другую невырожденную $(k, n)$-квадрику является рациональным отображением, степень которого (как числителя и знаменателя) равномерно ограничена единой константой $m$, зависящей лишь от $n$ и $k$. 
УТВЕРЖДЕНИЕ 6. Можно положить $m=6(n+k)$, т.е. если $Z \rightarrow R(Z, W) / T(Z, W), W \rightarrow S(Z, W) / T(Z, W)$ произвольное CR-отображение невырожденной $(k, n)$-квадрики в другую невырожденную $(k, n)$-квадрику, где $R=\left(R^{1}, \ldots, R^{n}\right), S=\left(S^{1}, \ldots, S^{k}\right), T$ - взаимно простые многочлены, то $\operatorname{deg} R^{p}, \operatorname{deg} S^{q}, \operatorname{deg} T$ не превосходят $6(n+k)$.

ДоказАТЕльство. Доказательство получается применением рассуждения Туманова с учетом того, что если $X=f \frac{\partial}{\partial z}+g \frac{\partial}{\partial w} \in g(Q)$, то $f$ и $g-$ многочлены степени не выше второй (см. (1.3)).

Эта оценка не является точной. При $k=1 m$ можно положить равньм 1 (см. [12]). При $k=2$ можно положить $m=2$ (см. [7], [10]). Наша гипотеза состоит в том, что всегда можно положить $m=k$.

В связи с этим вопросом можно сформулировать следующую задачу, которая является вполне независимой и на которую мне хотелось бы обратить внимание математической общественности.

Пусть $G$ - подгруппа групшы бирациональных автоморфизмов пространства $\mathbb{C}^{n}$ (групшы Кремоны), обладающая свойством равномерной ограниченности степени, т.е. сушествует постоянная $m$, такая, что для любого $g \in G$, где $g=(x \rightarrow A(x) / B(x))$ и дробь несократима, $\operatorname{deg} A \leqslant m$ и $\operatorname{deg} B \leqslant m$. Требуется дать описание таких групп $G$.

Задача описания таких групп не представляется безнадежной, так как, в некотором смысле, таких груп не должно быть. Действительно, при подстановке одного рационального отображения в другое степень должна возрастать. Имеюшиеся примеры - это исключения. Задача состоит в том, что их нужно явно описать. Ниже приводятся известные нам примеры.

\section{ПРИМЕР 5.}

(А) Дробно-линейные преобразования, т.е. автоморфизмы $\mathbb{C P}^{n}$

здесь $m=1$.

$$
x^{p} \rightarrow \frac{\sum a_{j}^{p} x^{j}}{\sum \alpha_{j} x^{j}},
$$

(В) Матрично дробно-линейные преобразования: $n=p^{2}, Z, A, B, C$, $D$ - матрицы размером $p \times p$. $Z \rightarrow(A Z+B) /(C Z+D)$, здесь $m=p$.

(С) Треугольные преобразования: $x \rightarrow x+f(y), y \rightarrow y$, где $f-$ произвольная рациональная функция, $m=\operatorname{deg} f$.

(D) Автоморфизмы невырожденных квадрик.

(E) Прямое произведение отображений пунктов (A)-(D) и они же, записанные в других координатах, бирационально связанных с исходными. 


\section{СПИСОК ЦИТИРОВАННОЙ ЛИТЕРАТУРЫ}

[1] Белошапка В. К. О построении нормальной формы уравнения поверхности высокой коразмерности // Матем. заметки. 1990. Т. 48. № 2. С. 3-9.

[2] Белошапка В. К. Теорема единственности для автоморфизмов невырожденной поверхности в комплексном пространстве // Матем. заметки. 1990. T. 47. № 3. C. $17-22$.

[3] Белошапка В. К. О голоморфных преобразованиях квадрики // Матем. сб. 1991. Т. 182 . № 2. С. 203-219.

[4] Белошапка В. K. Геометрические инварианты CR-многообразий // Maтем. заметки. 1994. Т. 55. № 5. С. 3-12.

[5] Шафаревич И. Р. Алгебраическая геометрия. М.: Изд-во МГУ, 1968.

[6] Лобода А. В. Порождающие вещественно аналитические многообразия коразмерности 2 в $\mathbb{C}^{2}$ и их биголоморфные отображения // Изв. АН СССР. Сер. матем. 1988. № 5. С. 970-990.

[7] Абросимов А. В. Описание локально биголоморфных автоморфизмов стандартных квадрик коразмерности два // Матем. сб. 1993. Т. 184. № 10. С. 3-52.

[8] Палинчак Н.Ф. О с-жестких квадриках // Матем. заметки. 1996. Т. 59. № 2 .

[9] Шевченко С.Н. Описание алгебры инфинитезимальных автоморфизмов квадрик коразмерности два и их классификация // Матем. заметки. 1994. T. 55. № 5. C. 142-153.

[10] Ezov V., Schmalz G. Holomorphic Automorphisms of Quadrics of Codimention 2. Preprint. Bohn: Max-Planck-Institut fur Mathematik, 1993.

[11] Туманов A.E. Конечность группы CR-автоморфизмов стандартного $\mathrm{CR}$-многообразия и собственные голоморфные отображения областей Зигеля // Изв. АН СССР. Сер. матем. 1988. Т. 52. № 3. С. 651-659.

[12] Chern S.S., Moser J. K. Real hypersurfaces in complex manifolds // Acta Math. 1974. V. 133. № 3-4. P. 219-271. 